\title{
Syrrx acquisition signals maturation of structure-based discovery
}

For the first time since incorporating 80 years ago, Takeda will open a drug discovery research site outside of Japan. To accomplish this, it is acquiring San Diego-based Syrrx, a high-throughput crystallography-based drug discovery firm, for $\$ 270$ million. Although Takeda's choice of a company with structure-based discovery capabilities is unlikely to indicate the start of a raft of acquisition activity in that industry segment, it suggests a maturation in the application of structural biology as a starting point for drug development.

Takeda had been talking to Syrrx for over a year about

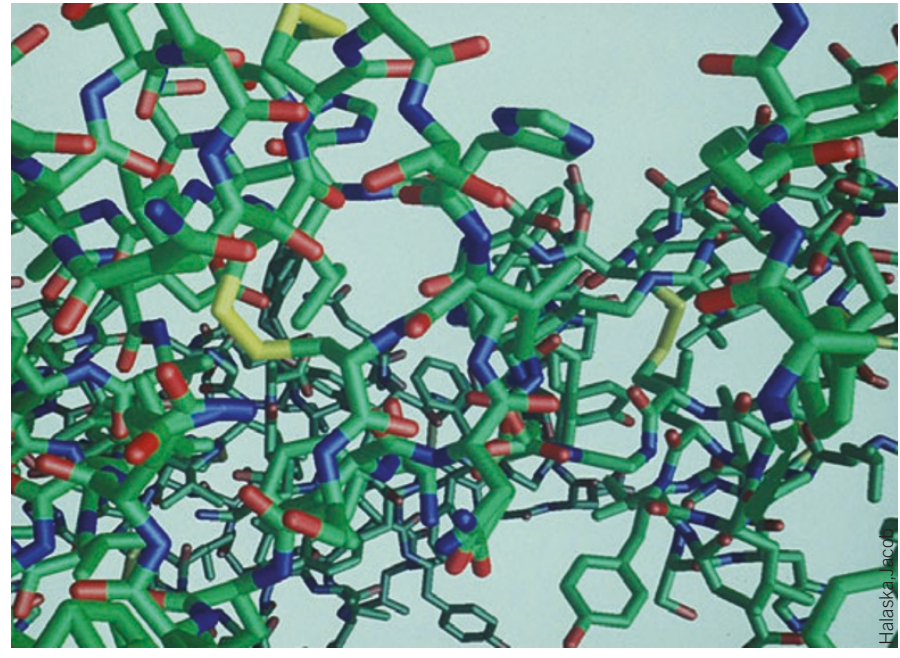

Structural biology is now used as a starting point for drug development.

ness model based on what's in vogue," observes Syrrx's Kaldor. "Today it's to follow the NRDO [no research, development only] model of only buying assets already in the clinic, and advancing them further. We were staring that trend in the face, but decided to continue to plow money into our own programs and take the risk of building a broad portfolio. We felt that with unique efficiency we could have our cake and eat it, too; that is, have tools and a pipeline."

Other players may follow a similar strategy, but because of Takeda's unique interests in the combination of Syrrx's technology, people and compounds, it may not lead to a similar exit. Like Syrrx, Plexxikon is trying of several of its metabolic disease and oncology programs - two disease areas of substantial interest to the Japanese company. In the course of those discussions, the deal expanded. "We saw the company had good IND [investigational new drug]-producing capabilities," says Tetsuo Miwa, director of the discovery research center at Takeda in Osaka.

Moreover, Takeda's researchers had an increasing demand for protein crystallization expertise, and although drug companies elsewhere were already using these technologies-Pfizer, GlaxoSmithKline, Eli Lilly and Johnson \& Johnson, for example, have either bought or built structure-based discovery capabilities-few scientists in Japan had the necessary automation experience in this area. "It would take time to set it up," Miwa explains.

"There's been an industrialization of the structural biology process," acknowledges Stephen Kaldor, Syrrx's president and CSO, driven by improvements in miniaturization, automation, parallel processing and data capture. As a result, Syrrx and other companies, notably Structural GenomiX (SGX), also in San Diego, and Berkeley, California's Plexxikon, are now using structural biology proactively, as a drug discovery tool. Until now, "the classic role of crystallography was retrospective," explains Michael Grey, CEO of SGX. "It was used to rationalize biological observations already made, rather than as a tool to make prospective decisions about what compounds to synthesize."

"Without this technology, chemists are more tempted to focus on the most potent

screening hits, rather than structurally diverse and drug-like hits with varying potencies," adds Takeda's Miwa. With the ability to solve a structure in months and rapidly obtain high-volume co-crystal data, chemists can see how a molecule binds to the protein target, which enables them to pursue leads that may not have strong bioactivity, but which can be optimized more easily. Moreover, $40-50 \%$ of the targets of current interest to large pharmas as well as biotechs_-including proteases, kinases and certain classes of membrane proteins-are amenable to a structure-based discovery approach.

With such broad potential, Syrrx, for its part, set out to be an IND-producing engine. It rapidly generated multiple clinical candidates for dipeptidyl peptidase 4 (DP4), a drug target for type 2 diabetes. But the cost of applying the platform repeatedly was daunting. "Think about the cost of two INDs per year," ponders company chairman Sam Colella, managing director of venture capital firm Versant Ventures, of Menlo Park in California. "If we stayed independent, we would have remained capital intensive." Alternatively, it could have tried an initial public offering (IPO). The question was whether Syrrx should continue to advance its programs into phase 3 , which would enhance its IPO valuation, or look for a near-term exit via an acquisition.

"It's interesting to talk about the extent to which privately held biotech companies are pressured to change their busi- to advance a broad portfolio of compounds: it brought an internally developed diabetes drug candidate PLX204, a peroxisome proliferator-activated receptor agonist, into phase 1, which it then partnered with Wyeth. SGX, on the other hand, is homing in on a niche market in a discrete area of oncology - the development of compounds to treat Gleevec (imatinib)-resistant chronic myelogenous leukemia-to give it a rapid route to commercialization. SGX has also joined the ranks of in-licensers, as a way to further integrate drug development into their business. Last July, it obtained Troxatyl (troxacitabine), a nucleoside analog for treating acute myelogenous leukemia, from Shire Pharmaceuticals. Meanwhile, Cambridge, UK-based Astex Technology, continues to provide services by applying its crystallography platform to targets provided by partners.

With big pharma focused on cost cutting, it's unlikely that other structure-based firms will find a comfortable exit through acquisition. And to manage a successful IPO, they will have to continue funding their programs through partnering. "Companies generally need robust phase 2 data to go public at a good valuation," notes SG Cowen's Papadopoulos. "Getting \$270 million from Takeda is a huge success."

Mark Ratner, Cambridge, Massachusetts

For more news and analysis go to news@nature.com www.nature.com/news 